\title{
Existence of solutions for nonlinear mixed type integro-differential functional evolution equations with nonlocal conditions
}

Shengli Xie*

"Correspondence: slxie@aiai.edu.cn Department of Mathematics and Physics, Anhui University of Architecture, Jinzhai Road, Hefei, Anhui 230022, P.R. China

\begin{abstract}
Using the Mönch fixed point theorem, this article proves the existence of mild solutions for nonlinear mixed type integro-differential functional evolution equations with nonlocal conditions in Banach spaces. Some restricted conditions on a priori estimation and measure of noncompactness estimation have been deleted, and compactness conditions of evolution operators or compactness conditions on a nonlinear term $f\left(t, X_{r}, X_{r}, X_{r}\right)$ have been weakened. Our results extend and improve many known results.
\end{abstract}

MSC: $34 \mathrm{G} 20 ; 34 \mathrm{~K} 30$

Keywords: integro-differential functional evolution equation; mild solution; nonlocal conditions; fixed point; Banach spaces

\section{Introduction}

Let $(X,\|\cdot\|)$ be a Banach space, $C[J, X]=\{x: J=[0, a] \rightarrow X, x(t)$ is continuous in $J\}$ with the norm $\|x\|_{C}=\sup _{t \in J}\|x(t)\|$. It is easy to verify that $C[J, X]$ is a Banach space. The space of $X$-valued Bochner integrable functions on $J$ with the norm $\|x\|_{1}=\int_{0}^{a}\|x(s)\| d s$ is denoted by $L[J, X]$. Consider the following nonlinear mixed type integro-differential functional evolution equations with nonlocal conditions in a Banach space $X(I V P)$,

$$
\begin{aligned}
& x^{\prime}(t)=A\left[x(t)+\int_{0}^{t} F(t-s) x(s) d s\right]+f\left(t, x\left(\sigma_{1}(t)\right),\left(K x_{\sigma_{2}}\right)(t),\left(H x_{\sigma_{3}}\right)(t)\right), \quad t \in J, \\
& x(0)=g(x)+x_{0},
\end{aligned}
$$

where

$$
\left(K x_{\sigma_{2}}\right)(t)=\int_{0}^{t} k\left(t, s, x\left(\sigma_{2}(s)\right)\right) d s, \quad\left(H x_{\sigma_{3}}\right)(t)=\int_{0}^{a} h\left(t, s, x\left(\sigma_{3}(s)\right)\right) d s,
$$

$A$ is the generator of a strongly continuous semigroup in the Banach space $X$, and $F(t)$ is a bounded operator for $t \in J, x_{0} \in X, f \in C\left[J \times X^{3}, X\right], g: C[J, X] \rightarrow X, k \in C[\triangle \times X, X]$, $\triangle=\{(t, s) \in J \times J: s \leq t\}, h \in C[J \times J \times X, X], \sigma_{i} \in C[J, J]$ and $\sigma_{i}(t) \leq t(i=1,2,3)$.

For the existence of mild solutions of integro-differential functional evolution equations in abstract spaces, there are many research results, see [1-16], and references therein. In order to obtain the existence and controllability of mild solutions in these study papers,

(c) 2012 Xie; licensee Springer. This is an Open Access article distributed under the terms of the Creative Commons Attribution License (http://creativecommons.org/licenses/by/2.0), which permits unrestricted use, distribution, and reproduction in any medium, provided the original work is properly cited. 
usually, some restricted conditions on a priori estimation and compactness conditions of an evolution operator or compactness conditions on $f\left(t, X_{r}, X_{r}, X_{r}\right)$ are used.

Recently, using a fixed point theorem, Haribhau Laxman Tidkey and Machindra Baburao Dhakne [1] have studied the existence of mild solutions of $I V P(1.1)-(1.2)$ when $\sigma_{i}(t)=t$ $(i=1,2,3)$, the compactness of the resolvent operator and the restricted condition

$$
M_{1}\left[\left\|x_{0}\right\|+G_{1}+L r b+L K r b^{2}+L K_{1} b^{2}+L H r b^{2}+L H_{1} b^{2}+L_{1} b\right] \leq r
$$

with $M_{1}\left[L b+L K b^{2}+L H b^{2}\right]<1$ is used. Malar [17] and Shi [18] studied the existence of mild solutions of semilinear mixed type integrodifferential evolution equations with the equicontinuous semigroup

$$
\left\{\begin{aligned}
x^{\prime}(t)= & A x(t)+f\left(t, x(t), \int_{0}^{t} a(t, s) k(s, x(s)) d s,\right. \\
& \left.\int_{0}^{a} b(t, s) h(s, x(s)) d s\right), \quad t \in[0, a], \\
x(0)= & x_{0}+g(x) .
\end{aligned}\right.
$$

Solvability of the scalar equation

$$
m(t)=K_{1}+K_{2} \int_{0}^{t} h(s, m(s), n(s), q(s)) d s, \quad t \in J
$$

and the restricted condition on measure of noncompactness estimation

$$
\int_{0}^{t}\left[\eta_{1}(s)+k_{1} \eta_{2}(s)+k_{2} \eta_{3}(s)\right] d s \leq K
$$

are used in [17]. But estimations (3.15) and (3.21) in [18] seem to be incorrect, as they have no meaning.

In this paper, using the Mönch fixed point theorem, we investigate the existence of mild solutions of IVP (1.1)-(1.2). Some restricted conditions on a priori estimation and measure of noncompactness estimation have been deleted, and compactness conditions of a resolvent operator or compactness conditions on a nonlinear term $f\left(t, X_{r}, X_{r}, X_{r}\right)$ have been weakened. Our results extend and improve some corresponding results in papers $[1-4$, 6-21].

\section{Preliminaries}

We will make the following assumptions:

$\left(H_{1}\right)$ A generates a strongly continuous semigroup in the Banach space $X$.

$\left(H_{2}\right) F(t) \in B(X), 0 \leq t \leq a . F(t): Y \rightarrow Y$ and for $x(\cdot)$ continuous in $Y, A F(\cdot) x(\cdot) \in L^{1}[J, X]$. For $x \in X, F^{\prime}(t) x$ is continuous in $t \in J$, where $B(X)$ is the space of all linear and bounded operators on $X$, and $Y$ is the Banach space formed from $D(A)$, the domain of $A$, endowed with the graph norm.

Definition 2.1 [5] $R(t)$ is a resolvent operator of (1.1) with $f \equiv 0$ if $R(t) \in B(X)$ for $0 \leq t \leq a$ and satisfies the following conditions:

(1) $R(0)=I$, the identity operator on $X$, 
(2) for all $x \in X, R(t) x$ is continuous for $0 \leq t \leq a$,

(3) $R(t) \in B(Y), 0 \leq t \leq a$; for $y \in Y, R(\cdot) y \in C^{1}[J, X] \cap C[J, Y]$ and

$$
\begin{aligned}
\frac{d}{d t} R(t) y & =A\left[R(t) y+\int_{0}^{t} F(t-s) R(s) y d s\right] \\
& =R(t) A y+\int_{0}^{t} R(t-s) A F(s) y d s, \quad 0 \leq t \leq a .
\end{aligned}
$$

The resolvent operator $R(t)$ is said to be equicontinuous if $\{t \rightarrow R(t) x: x \in B\}$ is equicontinuous for the entire bounded set $B \subset X$ and $t>0$. If $x \in C[J, X]$ satisfies the following integral equation:

$$
x(t)=R(t)\left(x_{0}+g(x)\right)+\int_{0}^{t} R(t-s) f\left(s, x\left(\sigma_{1}(s)\right),\left(K x_{\sigma_{2}}\right)(s),\left(H x_{\sigma_{3}}\right)(s)\right) d s, \quad t \in J,
$$

then $x$ is said to be a mild solution IVP (1.1)-(1.2).

Lemma 2.2 [14] Let the conditions $\left(H_{1}\right),\left(H_{2}\right)$ be satisfied. Then (1.1) with $f \equiv 0$ has a unique resolvent operator.

The following lemma is obvious.

Lemma 2.3 Let the resolvent operator $R(t)$ be equicontinuous. If there is $\rho \in L\left[J, \mathbb{R}^{+}\right]$such that $\|x(t)\| \leq \rho(t)$ for a.e. $t \in J$, then the set $\left\{\int_{0}^{t} R(t-s) x(s) d s\right\}$ is equicontinuous.

Lemma 2.4 [22] Let $V \in C[J, E]$ be an equicontinuous bounded subset. Then $\alpha(V(t)) \in$ $C\left[J, \mathbb{R}^{+}\right]\left(\mathbb{R}^{+}=[0, \infty)\right), \alpha(V)=\max _{t \in J} \alpha(V(t))$.

Lemma 2.5 [23] Let $V=\left\{x_{n}\right\} \subset L[J, E]$ and there exists $\sigma \in L\left[J, \mathbb{R}^{+}\right]$such that $\left\|x_{n}(t)\right\| \leq$ $\sigma(t)$ for any $x \in V$ and a.e. $t \in J$. Then $\alpha(V(t)) \in L\left[J, \mathbb{R}^{+}\right]$and

$$
\alpha\left(\left\{\int_{0}^{t} x_{n}(s) d s: n \in \mathbb{N}\right\}\right) \leq 2 \int_{0}^{t} \alpha(V(s)) d s, \quad t \in J
$$

Lemma 2.6 [24] (Mönch) Let E be a Banach space, $\Omega$ a closed convex subset in $E$ and $y_{0} \in \Omega$. Suppose that the continuous operator $F: \Omega \rightarrow \Omega$ has the following property:

$V \subset \Omega$ countable, $V \subset \overline{c o}\left(\left\{y_{0}\right\} \cup F(V)\right) \Rightarrow V$ is relatively compact.

Then $F$ has a fixed point in $\Omega$.

For $V \subset C[J, X]$, let $V(t)=\{x(t): x \in V\}, V_{\sigma_{i}}(t)=\left\{x\left(\sigma_{i}(t)\right): x \in V\right\}(i=1,2,3),(K V)(t)=$ $\{(K x)(t): x \in V\},(H V)(t)=\{(H x)(t): x \in V\}(t \in J), X_{r}=\{x \in X:\|x\| \leq r\}$ and $S_{r}=\{x \in$ $\left.C[J, X]:\|x\|_{C} \leq r\right\}$ for any $r>0 . \alpha(\cdot)$ and $\alpha_{C}(\cdot)$ denote the Kuratowski measure of noncompactness in $X$ and $C[J, X]$ respectively. For details on the properties of noncompact measure, we refer the reader to [22]. 


\section{Existence of a mild solution}

We make the following assumptions for convenience.

$\left(H_{3}\right)$ There exist constants $l_{g}>0, M>0$ and $4 l_{g} M<1$ such that

$$
\|g(x)-g(y)\| \leq l_{g}\|x-y\|_{C}, \quad x, y \in C[J, X]
$$

and $g(0)=0$.

$\left(H_{3}^{\prime}\right) g: C[J, X] \rightarrow E$ is continuous, compact and there exists a constant $N \geq 0$ such that $\|g(x)\| \leq N$.

$\left(H_{4}\right)$ There exists $q \in C\left[J, \mathbb{R}^{+}\right]$such that

$$
\|f(t, x, y, z)\| \leq q(t)(\|x\|+\|y\|+\|z\|), \quad t \in J, x, y, z \in X .
$$

$\left(H_{5}\right)$ There exist $k_{0} \in C\left[\triangle, \mathbb{R}^{+}\right], h_{0} \in C\left[J \times J, \mathbb{R}^{+}\right]$such that

$$
\begin{array}{cl}
\|k(t, s, x)\| \leq k_{0}(t, s)\|x\|, & (t, s) \in \triangle, x \in X, \\
\|h(t, s, x)\| \leq h_{0}(t, s)\|x\|, & t, s \in J, x \in X .
\end{array}
$$

$\left(H_{6}\right)$ For any $r>0$ and a bounded set $V_{i} \subset X_{r}$, there exist constants $l_{i}>0(i=1,2,3)$ such that

$$
\alpha\left(f\left(t, V_{1}, V_{2}, V_{3}\right)\right) \leq l_{1} \alpha\left(V_{1}\right)+l_{2} \alpha\left(V_{2}\right)+l_{3} \alpha\left(V_{3}\right), \quad t \in J
$$

$\left(H_{7}\right)$ For any $r>0$ and a bounded set $V \subset X_{r}$,

$$
\begin{array}{ll}
\alpha(k(t, s, V)) \leq k_{0}(t, s) \alpha(V), & (t, s) \in \Delta, \\
\alpha(h(t, s, V)) \leq h_{0}(t, s) \alpha(V), & t, s \in J .
\end{array}
$$

$\left(H_{8}\right)$ The resolvent operator $R(t)$ is equicontinuous and $\|R(t)\| \leq M e^{-w t}$ for $t \in J$ and some positive number

$$
w=\max \left\{2 M q_{0}\left(1+K_{0} a+H_{0} a\right), 4 M\left(l_{1}+2 l_{2} a K_{0}+2 l_{3} a H_{0}\right)\right\},
$$

where $K_{0}=\max _{(t, s) \in \Delta} k_{0}(t, s), H_{0}=\max _{t, s \in J} h_{0}(t, s), q_{0}=\max _{t \in J} q(t)$.

Without loss of generality, we always suppose that $x_{0}=0$.

Theorem 3.1 Let conditions $\left(H_{1}\right),\left(H_{2}\right),\left(H_{3}\right)-\left(H_{8}\right)$ be satisfied. Then IVP (1.1)-(1.2) has at least one mild solution.

\section{Proof Let}

$$
\begin{aligned}
(F x)(t)= & R(t) g(x) \\
& +\int_{0}^{t} R(t-s) f\left(s, x\left(\sigma_{1}(s)\right),\left(K x_{\sigma_{2}}\right)(s),\left(H x_{\sigma_{3}}\right)(s)\right) d s, \quad t \in J .
\end{aligned}
$$


We have by $\left(H_{3}\right),\left(H_{4}\right)$ and $\left(H_{5}\right)$,

$$
\begin{aligned}
& \|(F x)(t)\| \\
& \leq\|R(t) g(x)\|+\int_{0}^{t}\|R(t-s)\|\left\|f\left(s, x\left(\sigma_{1}(s)\right),\left(K x_{\sigma_{2}}\right)(s),\left(H x_{\sigma_{3}}\right)(s)\right)\right\| d s \\
& \leq M\|g(x)\|+M \int_{0}^{t} e^{-w(t-s)} q(s)\left(\left\|x\left(\sigma_{1}(s)\right)\right\|+\left\|(K x)\left(\sigma_{2}(s)\right)\right\|+\left\|(H x)\left(\sigma_{3}(s)\right)\right\|\right) d s \\
& \leq l_{g} M\|x\|_{C} \\
& \quad+M q_{0} \int_{0}^{t} e^{w(s-t)}\left(\|x(s)\|+\int_{0}^{s} k_{0}(s, r)\|x(r)\| d r+\int_{0}^{a} h_{0}(s, r)\|x(r)\| d r\right) d s \\
& \leq l_{g} M\|x\|_{C}+M q_{0} \int_{0}^{t} e^{w(s-t)}\left(1+K_{0} a+H_{0} a\right)\|x\|_{C} d s \\
& \leq l_{g} M\|x\|_{C}+M q_{0}\left(1+K_{0} a+H_{0} a\right) w^{-1}\|x\|_{C} \leq\|x\|_{C} .
\end{aligned}
$$

Let

$$
B_{R}=\left\{x \in C[J, X]:\|x\|_{C} \leq R\right\} .
$$

Then $B_{R}$ is a closed convex subset in $C[J, X], 0 \in B_{R}$ and $F: B_{R} \rightarrow B_{R}$. Similar to the proof of [6] and [9], it is easy to verify that $F$ is a continuous operator from $B_{R}$ into $B_{R}$. For $x \in B_{R}$, $s \in J,\left(H_{4}\right)$ and $\left(H_{5}\right)$ imply

$$
\left\|f\left(s, x\left(\sigma_{1}(s)\right),\left(K x_{\sigma_{2}}\right)(s),\left(H x_{\sigma_{3}}\right)(s)\right)\right\| \leq q(s)\left(1+\int_{0}^{s} k_{0}(s, r) d r+\int_{0}^{a} h_{0}(s, r) d r\right) R .
$$

We can show from (3.3), $\left(H_{8}\right)$ and Lemma 2.3 that $F\left(B_{R}\right)$ is an equicontinuous subset in $C[J, X]$.

Let $V \subset B_{R}$ be a countable set and $V \subset \overline{c o}(\{0\} \cup F(V))$, then

$$
V(t) \subset \overline{c o}(\{0\} \cup(F V)(t))
$$

From equicontinuity of $F\left(B_{R}\right)$ and (3.4), we know that $V$ is an equicontinuous subset in $C[J, X]$. By the properties of noncompact measure, the conditions $\left(H_{3}\right),\left(H_{6}\right),\left(H_{7}\right),(3.4)$ and Lemma 2.5, we have

$$
\begin{aligned}
\alpha(V(t)) \leq & \alpha((F V)(t)) \\
\leq & \|R(t)\| \alpha(g(V))+2 \int_{0}^{t}\|R(t-s)\| \alpha\left(f\left(s, V_{\sigma_{1}}(s),\left(K V_{\sigma_{2}}\right)(s),\left(H V_{\sigma_{3}}\right)(s)\right)\right) d s \\
\leq & l_{g} M \alpha_{C}(V)+2 M \int_{0}^{t} e^{w(s-t)}\left[l_{1} \alpha\left(V\left(\sigma_{1}(s)\right)\right)+2 l_{2} \int_{0}^{s} k_{0}(s, r) \alpha\left(V\left(\sigma_{2}(r)\right)\right) d r\right. \\
& \left.+2 l_{3} \int_{0}^{a} h_{0}(s, r) \alpha\left(V\left(\sigma_{3}(r)\right)\right) d r\right] d s \\
\leq & l_{g} M \alpha_{C}(V) \\
& +2 M \int_{0}^{t} e^{w(s-t)}\left[l_{1} \alpha(V(s)) d s+2\left(l_{2} \int_{0}^{s} K_{0}+l_{3} \int_{0}^{a} H_{0}\right) \alpha(V(r)) d r\right] d s
\end{aligned}
$$




$$
\begin{aligned}
& \leq l_{g} M \alpha_{C}(V)+2 M\left(l_{1}+2 l_{2} a K_{0}+2 l_{3} a H_{0}\right) \alpha_{C}(V) \int_{0}^{t} e^{w(s-t)} d s \\
& \leq l_{g} M \alpha_{C}(V)+2 M\left(l_{1}+2 l_{2} a K_{0}+2 l_{3} a H_{0}\right) w^{-1} \alpha_{C}(V) \leq \frac{3}{4} \alpha_{C}(V), \quad t \in J
\end{aligned}
$$

(3.5) together with Lemma 2.4 imply that $\alpha_{C}(V) \leq \frac{3}{4} \alpha_{C}(V)$, and so $\alpha_{C}(V)=0$. Hence $V$ is relatively compact in $C[J, X]$. Lemma 2.6 implies that $F$ has a fixed point in $C[J, X]$. Then IVP (1.1)-(1.2) has at least one mild solution. The proof is completed.

Theorem 3.2 Let the conditions $\left(H_{1}\right),\left(H_{2}\right)$ and $\left(H_{3}^{\prime}\right)-\left(H_{8}\right)$ be satisfied. Then IVP (1.1)-(1.2) has at least one mild solution.

Proof Similar to (3.2) and (3.5), it is easy to verify

$$
\|(F x)(t)\| \leq M N+M q_{0}\left(1+K_{0} a+H_{0} a\right) w^{-1}\|x\|_{C}=M N+\eta\|x\|_{C},
$$

where $\eta=M q_{0}\left(1+K_{0} a+H_{0} a\right) w^{-1}<1$. Taking $R>M N(1-\eta)^{-1}$, let $B_{R}=\left\{x \in C[J, X]:\|x\|_{C} \leq\right.$ $R$ \}. We have $F: B_{R} \rightarrow B_{R}$ and the inequality (3.5) is transformed into $\alpha(V(t)) \leq \frac{1}{2} \alpha_{C}(V)$, $t \in J$.

The other proof is similar to the proof of Theorem 3.1, we omit it.

\section{An example}

Let $X=L^{2}[0, \pi]$. Consider the following partial functional integro-differential equation with a nonlocal condition,

$$
\left\{\begin{aligned}
u_{t}(t, y)= & u_{y}(t, y)+\int_{0}^{t} F(t-s) u_{y}(s, y) d s+\gamma_{1} \sin u(t-r, y) \\
& +\int_{0}^{t} \frac{\gamma_{2} u(s-r, y) d s}{(1+t)}+\int_{0}^{a} \frac{\gamma_{3} u(s-r, y) d s}{(1+t)(1+s)^{2}}, \quad 0 \leq t \leq a \\
u(0, y)= & u_{0}(y)+\gamma_{4} u(y)
\end{aligned}\right.
$$

where $r, \gamma_{i} \in \mathbb{R}(i=1,2,3,4), \sigma_{1}(t)=\sigma_{2}(t)=\sigma_{3}(t)=t-r, 0 \leq r \leq t \leq a, F(t)$ satisfies the condition $\left(H_{2}\right)$,

$$
\begin{aligned}
& f\left(t, u(\sigma(t)),\left(K u_{\sigma}\right)(t),\left(S u_{\sigma}\right)(t)\right)(y) \\
& \quad=\gamma_{1} \sin u(t-r, y)+\int_{0}^{t} \frac{\gamma_{2} u(s-r, y) d s}{(1+t)}+\int_{0}^{a} \frac{\gamma_{3} u(s-r, y) d s}{(1+t)(1+s)^{2}}, \\
& k(t, s, u(\sigma(s)))(y)=\frac{u(s-r, y)}{1+t}, \quad h(t, s, u(\sigma(s)))(y)=\frac{u(s-r, y)}{(1+t)(1+s)^{2}}, \\
& g(u)(y)=\gamma_{4} u(y) .
\end{aligned}
$$

Let the operator $A$ be defined by $A w=w^{\prime}, w \in D(A)$ with the domain

$$
D(A)=\left\{w \in E: w^{\prime} \in E, w^{\prime} \text { is almost everywhere bounded }\right\} .
$$

Then $A$ generates a translation semigroup $R(t)$ and $R(t)$ is equicontinuous. The problem (4.1) can be regarded as a form of IVP (1.1)-(1.2). We have by (4.2), (4.3) and (4.4),

$$
\|f(t, u, v, z)\| \leq|\gamma|(\|u\|+\|v\|+\|z\|), \quad|\gamma|=\max \left\{\left|\gamma_{1}\right|,\left|\gamma_{2}\right|,\left|\gamma_{3}\right|\right\}, u, v, z \in X
$$




$$
\|k(t, s, u)\| \leq\|u\|, \quad\|h(t, s, u)\| \leq\|u\|, \quad u \in X,
$$

and

$$
\|g(u)-g(v)\| \leq\left|\gamma_{4}\right|\|u-v\|_{C}, \quad g(0)=0 .
$$

$\gamma_{4}$ and $M$ can be chosen such that $4 M\left|\gamma_{4}\right|<1$. In addition, for any $r>0$ and a bounded set $V_{i} \subset X_{r}(i=1,2,3)$, we can show that by the diagonal method,

$$
\begin{aligned}
& \alpha\left(f\left(t, V_{1}, V_{2}, V_{3}\right)\right) \leq|\gamma|\left(\alpha\left(V_{1}\right)+\alpha\left(V_{2}\right)+\alpha\left(V_{3}\right)\right), \quad t \in J, \\
& \alpha\left(k\left(t, s, V_{1}\right)\right) \leq \alpha\left(V_{1}\right), \quad t, s \in \triangle, \\
& \alpha\left(h\left(t, s, V_{1}\right)\right) \leq \alpha\left(V_{1}\right), \quad t, s \in[0, a] .
\end{aligned}
$$

Hence all the conditions of Theorem 3.1 are satisfied, the problem (4.1) has at least one mild solution in $C[J, X]$.

\section{Competing interests}

The author declares that they have no competing interests.

\section{Acknowledgements}

The work was supported by Natural Science Foundation of Anhui Province (11040606M01) and Education Department of Anhui (KJ2011A061, KJ2011Z057), China.

Received: 5 May 2012 Accepted: 22 August 2012 Published: 7 September 2012

\section{References}

1. Tidke, HL, Dhakne, MB: Existence of solutions and controllability of nonlinear mixed integrodifferential equation with nonlocal conditions. Appl. Math. E-Notes 11, 12-22 (2011)

2. Yan, Z, Wei, P: Existence of solutions for nonlinear functional integrodifferential evolution equations with nonlocal conditions. Aequ. Math. 79, 213-228 (2010)

3. Tidke, HL, Dhakne, MB: Nonlocal Cauchy problems for nonlinear mixed integro-differential equations. Tamkang J. Math. 41, 361-373 (2010)

4. Balachandran, K, Kumar, RR: Existence of solutions of integrodifferential evolution equations with time varying delays. Appl. Math. Lett. 7, 1-8 (2007)

5. Kumar, RR: Nonlocal Cauchy problem for analytic resolvent integrodifferential equations in Banach spaces. Appl. Math. Comput. 204, 352-362 (2008)

6. Yang, YL, Wang, JR: On some existence results of mild solutions for nonlocal integro-differential Cauchy problems in Banach spaces. Opusc. Math. 31, 443-455 (2011)

7. Liu, Q, Yuan, R: Existence of mild solutions for semilinear evolution equations with non-local initial conditions. Nonlinear Anal. 71, 4177-4184 (2009)

8. Boucherif, A, Precup, R: Semilinear evolution equations with nonlocal initial conditions. Dyn. Syst. Appl. 16, 507-516 (2007)

9. Chalishajar, DN: Controllability of mixed type Volterra-Fredholm integro-differential systems in Banach space. J. Franklin Inst. 344, 12-21 (2007)

10. Xue, X: Existence of solutions of a semilinear nonlocal Cauchy problem in a Banach space. Elect. J. Diff. Eqs. 2005(64), $1-7(2005)$

11. Liu, JH, Ezzinbi, K: Non-autonomous integrodifferential equations with nonlocal conditions. J. Integral Equ. Appl. 15 79-93 (2003)

12. Ntouyas, S, Tsamotas, P: Global existence for semilinear evolution equations with nonlocal conditions. J. Math. Anal. Appl. 210, 679-687 (1997)

13. Byszewski, L, Akca, H: Existence of solutions of a semilinear functional-differential evolution nonlocal problem. Nonlinear Anal. 34, 65-72 (1998)

14. Lin, Y, Liu, J: Semilinear integrodifferential equations with nonlocal Cauchy problem. Nonlinear Anal. 26, 1023-1033 (1996)

15. Byszewski, L: Existence and uniqueness of solutions of semilinear evolution nonlocal Cauchy problem. Zesz. Nauk. Politech. Rzesz., Mat. Fiz. 18, 109-112 (1993)

16. Byszewski, L: Theorems about the existence and uniqueness of solutions of a semilinear evolution nonlocal Cauchy problem. J. Math. Anal. Appl. 162, 494-505 (1991)

17. Malar, K: Existence of mild solutions for nonlocal integro-differential equations with measure of noncompactness. Int. J. Math. Sci. Comput. 1, 86-91 (2011)

18. Shi, H, Li, W, Sun, H-R: Existence of mild solutions for abstract mixed type semilinear evolution equations. Turk. J. Math. 35, 457-472 (2011) 
19. Zhu, T, Song, C, Li, G: Existence of mild solutions for abstract semilinear evolution equations in Banach spaces. Nonlinear Anal. 75, 177-181 (2012)

20. Fan, Z, Dong, Q, Li, G: Semilinear differential equations with nonlocal conditions in Banach spaces. Int. J. Nonlinear Sci. 2(3), 131-139 (2006)

21. Dong, Q, Li, G: Existence of solutions for semilinear differential equations with nonlocal conditions in Banach spaces. Electron. J. Qual. Theory Differ. Equ. 47, 1-13 (2009)

22. Dajun, G, Lakshmikantham, V, Liu, X: Nonlinear Integral Equations in Abstract Spaces. Kluwer Academic, Dordrecht (1996)

23. Heine, HP: On the behavior of measure of noncompactness with respect to differentiation and integration of vector valued functions. Nonlinear Anal. 7, 1351-1371 (1983)

24. Mönch, $\mathrm{H}$ : Boundary value problems for nonlinear ordinary equations of second order in Banach spaces. Nonlinear Anal. 4, 985-999 (1980)

doi:10.1186/1687-2770-2012-100

Cite this article as: Xie: Existence of solutions for nonlinear mixed type integro-differential functional evolution equations with nonlocal conditions. Boundary Value Problems 2012 2012:100.

Submit your manuscript to a SpringerOpen ${ }^{\circ}$ journal and benefit from:

- Convenient online submission

- Rigorous peer review

- Immediate publication on acceptance

- Open access: articles freely available online

- High visibility within the field

- Retaining the copyright to your article

Submit your next manuscript at $>$ springeropen.com 